\title{
INVESTIGACIÓN/RESEARCH
}

Recibido: 02/09/2012---Revisado: 02/11/2012 Aceptado: 03/01/2012---Publicado: 15/07/2013

\section{ANÁLISIS DE MANUALES DE URBANIDAD COMO MEDIO DE COMUNICACIÓN: \\ NOVÍSIMO RESUMEN DE URBANIDAD PARA NIÑAS DE DON NARCISO MASDIVAL PUIG}

Andreína Victoria Sevilla Gutiérrez: Universidad Metropolitana. (Venezuela) Andreinasevilla1@gmail.com

\section{Resumen}

El siguiente artículo analiza cómo los Manuales de Urbanidad establecían los lineamientos de comportamiento para los jóvenes y sus efectos en el modelo educativo imperante. En el siguiente análisis se revisa el período comprendido entre 1902 a 1931 centrándonos en el Manual de Narciso Masdival Puig (1927) el cual realiza una guía donde manifiesta que las jóvenes tienen una misión a la cual deben estar apegadas para el desarrollo de la vida en sociedad. De esto se desprenden las bases fundamentales para la formación de las niñas, la cual determinará las acciones que desempeñen dentro de la comunidad y las limitaciones para su desarrollo intelectual en el ámbito académico y profesional.

Palabras clave: Manual de Urbanidad - Lineamientos de ComportamientoDesarrollo Intelectual - Modelo Educativo - Sociedad.

\footnotetext{
${ }^{1}$ Autor correspondiente:

Andreína Victoria Sevilla Gutiérrez: Procedente de la Universidad Metropolitana, sita en Caracas, Venezuela.

Correo: andreinasevilla1@gmail.com
} 


\title{
ANALYSIS URBANITY MANUALS AS MASS MEDIA: NEWEST SUMMARY URBANITY FOR GIRLS OF DON NARCISO MASDIVAL PUIG
}

\begin{abstract}
The civility manuals established behavioral guidelines for young people. The following analysis outlines the period between 1902-1931, focusing on the manual of Narciso Masdival Puig (1927) that is a guide that states the mission young woman have to the development of social life. This gives rise to the fundamental basis for the training of girls, which determine the actions you perform within the community and limitations to intellectual development in academic and professional.
\end{abstract}

Key words: Manual of Civility - Behavior Guidelines-Intellectual Development - Model Education - Society.

\section{INTRODUCCIÓN}

Los Manuales de Urbanidad ofrecen los lineamientos de los valores, los principios y el comportamiento social que deben tener los jóvenes. Para efectos del análisis a realizar, limitaremos el periodo histórico entre 1902 a 1931 y nos enfocaremos en la Urbanidad para las niñas utilizando el Novísimo Resumen de Urbanidad para Niñas de Don Narciso Masdival Puig (1927) una guía que describe como es "el deber ser" de las jovencitas para conducirse en la sociedad y convertirse en mujeres útiles en el futuro.

El Resumen está dividido en varias secciones dedicadas a la formación educacional de las jovencitas en diferentes esferas de la vida. En el primer apartado, Puig (1927) realiza una introducción bosquejando el carácter qué deben gozar las niñas, luego continúa explicando el significado de urbanidad y determinando como deben dirigir sus acciones para la comunidad. En el tercer apartado, prosigue señalando los deberes de las niñas con la religiosidad y su comportamiento en las iglesias, posteriormente realiza una guía de comportamiento para la estancia en el colegio y su manejo ante las visita sociales; para finalizar, establece los tratamientos para las personas importantes y describe cómo deben dirigirse a las personas de renombre.

\section{METODOLOGÍA}

Para realizar el análisis, centraremos la investigación en la categoría de la educación de las niñas en el mundo académico, es decir, su papel dentro de la formación profesional y su privación para el desarrollo intelectual de las ciencias.

Se trata de un trabajo historiográfico que busca decantar los efectos de los Manuales de Urbanidad en la educación de las jóvenes y su efecto como un medio, para la propagación de un mandato que establece como debe ser la actuación de las mujeres en la sociedad a través de una metodología bibliográfica, que se propone analizar 
Manual de Urbanidad - Lineamientos de Comportamiento- Desarrollo Intelectual Modelo Educativo - Sociedad

hasta que punto el modelo educativo imperante en una época puede constituirse como un objeto de limitación para el desarrollo intelectual.

\section{ANÁLISIS Y DISCUSIÓN}

\subsection{Breve análisis del manual de urbanidad para niñas (1927)}

La concepción de urbanidad para la época estudiada es definida según el Manual como "El arte de arreglar nuestras acciones y conducirnos con buen modo en la sociedad." (Puig, 1927, p.9). Será este modelo el que predominará en la educación para los jóvenes, ya que, "las buenas maneras forman parte del currículo escolar durante el siglo XIX y parte importante del XX..." (Ampudia, 2004, p.264). De esta manera, se transmiten las instrucciones a las que deben atender las niñas para ser las mujeres que desea la sociedad y cuáles son las formas y el comportamiento que éstas deben seguir para lograrlo.

Para ello, las niñas deben tener una buena educación en los medios físicos y morales; es decir deben gozar de aseo, finura, comedimiento, esbeltez y hermosura, además de actuar bajo las virtudes cristianas pues de esta forma producen honradez a la familia y la remodelación de la sociedad. (Puig, 1927, p.1-2). Razones por las cuales los modelos de escolarización para las niñas estaban dirigidos a las "labores de manos" como coser, hilar y bordar; actividades conformes al rol de la mujer en sociedad. (Sarasúa, 2002, p.287)

Masdival Puig (1927) manifiesta que las niñas tienen una misión determinada en la vida del hogar y la familia, señalando que éstas no están

"Destinadas a gobernar un Estado, ni a ir a la guerra, ni a las Academias y Parlamentos, ni ejercer el ministerio de la Iglesia; por consiguiente, [deben] prescindir de las ciencias políticas, del arte militar, de la Jurisprudencia y de la Teología." (Puig, 1927, p.7)

Esta afirmación manifiesta la condición de subordinación que tenían las mujeres en el área de formación profesional, pues, solo debían atender los deberes del hogar y dejar a los hombres el conocimiento de las ciencias. Se trata entonces de la convicción de que "la educación femenina era ante todo un asunto moral y por lo tanto familiar, siendo más importante desde luego la "educación" (la transmisión de valores morales y religiosos) que la "instrucción" (el acceso a determinados saberes reglados)." (Guereña, sf, p.26).

La educación de las niñas se centra en la casa y la iglesia manifestando una sociedad con una marcada distinción de sexo, con determinados espacios donde cada cual debe actuar dentro de sus esferas de acción, que como consecuencia conlleva a una sociedad intelectualmente dirigida por hombres. Las mujeres deben limitarse a ser esposas y madres, que es la misión de las niñas y deben formarse para eso. 


\subsection{Efectos de la aplicación de los manuales de urbanidad como modelo educativo}

Como resultado de esta discriminación intelectual encontramos altos índices de analfabetismo en niñas en comparación con los varones durante los años que comprende el estudio: Así, en España, para 1900 el $71 \%$ de las mujeres era analfabeta contra el $56 \%$ de los varones. (Liébana, 2009, p.5). Vemos entonces que la estructura educacional modelaba las enseñanzas de cada género, determinando así que las niñas eran preparadas para los quehaceres del hogar y los niños se instruían en las ciencias y los conocimientos. El problema no estaba en si existían las escuelas de niñas sino que dichas escuelas convivían con el analfabetismo por no tener interés de enseñar la escritura u otras ramas del conocimiento a las jovencitas. (Sarasúa, 2002, p. 286).

La Ley Moyano había declarado que era obligatorio "la creación de escuelas de niñas en pueblos de más de 500 almas"(Scalon, s.f, p.194). Sin embargo, estas escuelas no garantizaban una enseñanza óptima de las jóvenes, ya que, "parte de un concepto de la educación femenina en función de la tradicional división de trabajo entre los dos sexos." (Scalon, s.f, p.194) y minimiza la enseñanza de la mujer a ser esposa y madre. Esta condición era promovida por las familias, donde

"Los padres que escolarizaban a sus hijas no querían
necesariamente para éstas la lectura y la escritura, mucho menos
la Gramática, la Historia o la Aritmética: lo que buscaba la
enseñanza que se daba a las niñas no era prepararlas para
<<empleos>> como los de los hombres, sino para lo que la
sociedad consideraba su función natural: llevar una casa, criar a
sus hijos, ensenarles a rezar, cocinar, cuidar a su marido, coser,
hacer media, hilar y tejer, quizá hacer encaje, bordar y remendar."
(Sarasúa, 2002, p. 286)

En esta cita evidenciamos la división que existía entre la enseñanza que se impartia a hombres y a mujeres, aunado a una convicción compartida por diferentes actores de la vida que le atribuye una inferioridad a la capacidad de la mujer, disminuyendo su papel dentro de la sociedad, como señala Pilar Ballarín (2007) catedrática de la Universidad de Granada:

"Durante el siglo XIX español, el papel doméstico de las mujeres que iba definiéndose en el pensamiento liberal se aproximaba mucho al de la cultura católica[...] En la búsqueda de ganar posiciones a través de las mujeres vinieron a coincidir el poder político y el religioso. En ambos casos, confinar a las mujeres al espacio doméstico constituía un bien social y moral. El modelo liberal burgués las alejaría de lo político, del espacio público y del mundo laboral convirtiéndolas, como madres y esposas que gobiernan el hogar, en el soporte sobre el que construir la nueva 
ciudadanía de los varones. El modelo católico, que frente a la creciente secularización buscaba la recatolización a través de las mujeres, no tendría más que exculparlas del pecado original y convertir en compañeras a quienes venían siendo esclavas, para seguir manteniéndolas en su papel."(Ballarín, 2007, p.146)"

Entonces, podemos apreciar el interés por parte del Estado de mantener una instrucción pedagógica de las niñas favorable para el mantenimiento del statu quo, pues era cuestión de intereses sociales y económicos, ya que "la tradición en las sociedades distribuye a las mujeres la porción más pequeña del producto social. Pero sin importar si el producto final de la tradición va de acuerdo o no con nuestra perspectiva moral, debemos entender que es un método práctico de dividir la producción de la sociedad" (Milberg y Heilbroner, s.f, p.8) en este caso, y de conformidad con la cita precedente, se trata de la producción del elemento cohesionador de la moral y la familia que acaecen en bienes a la sociedad y proporcionan estabilidad al funcionamiento de la comunidad.

Por otra parte, también debemos considerar para este análisis y contribuir a la discusión sobre el punto estudiado, la influencia que ejercía la iglesia en el sistema educativo de las niñas y como la institución religiosa colaboraba en el mantenimiento del papel secundario de la mujer en la sociedad, limitándola al sector domestico. A través de una enseñanza netamente discriminatoria dirigida a formar a las jovencitas en las labores del hogar y la moral, actividades propias de su sexo. Como señala el Manual de Narciso Masdival (1927) este es "aprobado por la Autoridad eclesiástica" manifestando su conformidad con la educación inferior que daban los manuales escolares a las jóvenes.

De esta forma, se destaca el interés de las actores sociales por el mantenimiento de un rol limitado del sexo femenino, pues se trata de un "modelo de mujer dedicada a las labores de manos en el espacio domestico, un modelo moral y económico que había condicionado la enseñanza" (Sarasúa, 2002, p.296) para el provecho e interés del Estado y la Iglesia.

El sistema educativo de este periodo se centra entonces en la búsqueda de los provechos sociales desviando de esta forma la verdadera enseñanza para el desarrollo intelectual, social y personal; pues

"A lo largo de este período «de furor y de ruido» que va de 1914 a, digamos, 1989, el debate educativo ha tendido a marginar o dejar en segundo plano el tema inicial de la relación entre la educación y el orden de libertad, para centrarse en otros temas, sin duda de cierta importancia pero relativamente secundarios, como son los de hacer que la educación fuera un instrumento para otros objetivos, y de este modo asegurarse de la contribución de la educación al funcionamiento de la economía, a la eventual grandeza de las naciones en el concierto mundial, o al reforzamiento de su cohesión social (por los procedimientos 
diversos de legitimar sus jerarquías o reducir sus desigualdades, de homogeneizar sus poblaciones o, por el contrario, afianzar su diversidad)."(Díaz, 2001, p.15-16)

La afirmación que se desprende de la cita anterior refleja el carácter meramente instrumental de la enseñanza en las niñas para lograr ciertos objetivos sociales y no para el crecimiento intelectual. No se trataba de su educación sino del cumplimiento de una misión discriminatoria designada por otros actores de la vida social que cercenan la libertad, la capacidad de elección y el determinismo de las niñas.

\title{
3.3 Aportes para la igualdad de la mujer
}

El Manual de Urbanidad manifiesta la creencia de una sociedad al servicio de imperativos morales que discriminan a la mujer. No obstante, una parte de la sociedad no lo aceptaba tan mansamente pues se pueden apreciar antecedentes en obras en las que se proclamaba la igualdad de las mujeres, como la obra de Benito Feijoo un representante de la ilustración española, donde manifiesta "que mujeres y hombres «son iguales en cuanto que seres racionales, pues la razón no tiene sexo». Reconocía que era la desigualdad en la educación la que impedía que se desarrollaran sus potencialidades." (Ballarin, s.f, p. 146). De esta manera, Campomanes "crítico de la educación conventual, habitual en la educación de las niñas; propuso la creación de colegios laicos para la enseñanza de las jovencitas, en los que se impartiría gramática, francés, historia, geografía, baile, nociones de música y poesía." (Gallego, s.f, p.85). Es decir,

\begin{abstract}
"El pensamiento ilustrado entendió la cuestión de la educación de la mujer como un elemento más del engranaje nacional, encajado en la reforma y dirigido al progreso del país; pero las mujeres no salían de sus tradicionales competencias maternales y domésticas." (Gallego, s.f, p.84)
\end{abstract}

Por otra parte, el caso de dos mujeres que ya para 1880 tenían presencia en las facultades universitarias demostrando el carácter transformador social que podía dar el hecho de la inclusión femenina en los modelos de educación. "Esas dos primeras doctoras fueron Dolores Aleu Riera y Martina Castells Ballespi" (García, 1998, p.244) que manifestaron la igualdad en inteligencia de los géneros y la importancia de aumentar el nivel de estudio de las mujeres para el desarrollo de la humanidad. A pesar de esto, no fue un hecho que cambió significativamente la idea social de la inferioridad de la mujer, pues

"Que la mujer se educara no sólo como el hombre sino con el hombre era sin duda a principios de siglo una aspiración del todo ilusoria. Sin embargo, en los años siguientes los Institucionistas trabajaron para acortar la distancia entre la instrucción femenina y la masculina, influyendo en la política pedagógica de los ministerios liberales." (Scalon, s.f, 202) 
Manual de Urbanidad - Lineamientos de Comportamiento- Desarrollo Intelectual Modelo Educativo - Sociedad

Ya para 1927 cuando es publicado el Manual de Masdival (1927) las instituciones habían realizado un gran avance en la nivelación educacional de las niñas para convertirlas en mujeres activas y profesionales de ciertas áreas, ampliando su cultura y concediéndoles espacios para la participación en Facultades Universitarias, en la Escuela Superior del Magisterio, en las bibliotecas y laboratorios. Los niveles de analfabetismo se redujeron y los de escolarización aumentaron significativamente. (Scalon, s.f, 205-206).

\section{CONCLUSIONES}

En la sociedad del siglo XX existía una desviación entre el hecho y el derecho, pues si bien se habían realizado numerosos avances en la educación de la mujer para la fecha, éstos convivían con la convicción tradicional de la misión de las mujeres destinadas a ser amas de casa, como se evidencia en el Manual de Narciso Masdival Puig (1927).

Se puede concluir de lo antes expuesto que el sistema educativo, fundamentado en los Manuales de Urbanidad durante este período se centran en la formación de la mujer para un fin instrumental, se pierde de vista la formación de la persona y su enriquecimiento individual para que impere la convicción tradicional del papel de la mujer limitada a los labores del hogar, lo que conlleva a una desigualdad en el acceso a la formación académica y profesional de las mujeres.

\section{BIBLIOGRAFÍA}

Ampudia de Haro, Fernando (2004): "La civilización del comportamiento: urbanidad y buenas maneras en España desde la Baja Edad hasta nuestros días" Universidad Complutense de Madrid. Disponible en: http://eprints.ucm.es/10497/1/T28072.pdf (Consultado el 22/06/2013)

Ballarín Domingo, Pilar (2007): "La escuela de niñas en el siglo XIX: la legitimación de la sociedad de esferas separadas" en Revista Universidad de Granada. Disponible

en: http://campus.usal.es/ revistas_trabajo/index.php/02120267/article/viewFile/743/917 (Consultado el 22/06/2013)

Pérez-Díaz, Víctor, Rodríguez, Juan y Ferrer, Leonardo (2001): "La familia española ante la educación de sus hijos" Fundación la Caixa. Colección de estudios sociales. Núm.

5.

Disponible

en:

http://obrasocial.lacaixa.es/StaticFiles/StaticFiles/f0e90d9e7c8ef010VgnVCM1000000e 8cf10aRCRD/es/es05_esp.pdf (Consultado el 23/06/2013)

Gallego, Elena (s.f): "La educación de las mujeres en los discursos ilustrados" en Publicación Colección Mediterráneo Económico. Núm. 9. Disponible en: http://www.publicacionescajamar.es/pdf/publicaciones-periodicas/mediterraneoeconomico/9/9-131.pdf (Consultado el 23/06/2013) 
García Flecha, Consuelo (1999): "La educación de la mujer según las primeras doctoras en medicina de la Universidad española, año 1882" en DYNAMIS. Disponible en:

http://www.ugr.es/ dynamis/completo19/PDF/12.\%20La\%20educacion\%20de\%20la \%20mujer.pdf (Consultado el 22/06/2013)

Guereña, Jean-Louis (s.f): "Mujeres autoras de libros de texto. El caso de los manuales de urbanidad en la segunda mitad del siglo XIX y principios del siglo XX" en Lectures du genre no 2: Femmes/Histoire/histoires. Disponible en: http://www.lecturesdugenre.fr/Lectures_du_genre_2/Guerena_files/GUERENA.pdf (Consultado el 23/06/2013)

Liébana, Alfredo (2009): "La educación en España en el primer tercio del siglo XX: la situación del analfabetismo y la escolarización" Madrid: Universidad de Mayores de Experiencia Recíproca. Disponible en: http://www.umer.es/images/doc/n58.pdf (Consultado el 22/06/2013)

Milberg, William, HEILBRONER, Robert $(s, f)$ : La evolución de la sociedad económica. Decima edición. México: Editorial Pearson. Disponible en: http://es.scribd.com/doc/142297906/Heilbroner-Milberg-Evolucion-de-la-sociedadeconomica (Consultado el 23/06/2013)

Puig, Narciso (1927): "Novísimo Resumen de Urbanidad para niñas" Librería ROSALS: Barcelona. Disponible en: http://www.uned.es/manesvirtual/BibliotecaManes/FormCivica/FCiv1902_31/ResMas/R esMas01.html (Consultado el 22/06/2013)

Sarasúa, Carmen (2002) "Aprendiendo a ser mujeres: las escuelas de niñas en la España del siglo XIX" en Revista Científica Universidad Complutense Madrid. Cuadernos de Historia Contemporánea. Vol 24. Disponible en: http://revistas.ucm.es/index.php/CHCO/article/view/CHCO0202110281A/6926 (Consultado el 23/06/2013)

Scanlon, Geraldine (s,f): "La mujer y la instrucción pública: de La Ley de Moyano a la II República" en Repositorio Documental de la Universidad de Salamanca. Disponible en: http://gredos.usal.es/jspui/bitstream/10366/79441/1/La_mujer_y_la_instruccion_publi ca_de_la_.pdf (Consultado el 23/06/2013)

\section{Andreina Victoria Sevilla Gutiérrez}

Procede de la Universidad Metropolitana de Venezuela, sita en Caracas. 